\title{
Evidence for the effectiveness of anti- hypertensive medicines included on the Chinese National Reimbursement Drug List
}

\author{
Wenbin Liu', Lizheng Shi ${ }^{2}$, Monika Sawhney ${ }^{3}$, Xiaoli Gu ${ }^{4}$ and Yingyao Chen ${ }^{5^{*}}$
}

\begin{abstract}
Background: Evidence-based decision on drug list or formulary has been applied worldwide. Although the importance of scientific evidence was emphasized, the decision-making procedures for including medicines into the national reimbursement drug list were often challenged by their process opacity and relying on subjective expert opinion. This study aimed to explore and assess the evidence for the effectiveness of antihypertensive medicines included on the Chinese National Reimbursement Drug List (NRDL), and to provide recommendations for further improvement.

Methods: Three international evidence-based guidelines were selected to serve as reference criteria. The antihypertensive medicines included in NRDL of Urban Employee Basic Medical Insurance (UEBMI) were compared with recommended drugs in three international guidelines. Medicines recommended by at least two guidelines were considered to have sound evidence support for the effectiveness. Otherwise, published literature with high evidence grade, namely systematic review, meta-analysis and randomized controlled trial $(\mathrm{RCT})$, were searched for further assessment. Medicines reported as fairly good effectiveness by literature with high evidence grade can be also considered having sound evidence for the effectiveness. Methodological quality of systematic review or meta-analysis was evaluated by AMSTAR scale and PRISMA statement. Literature quality of RCTs was assessed by Jadad scale.
\end{abstract}

Results: For the 97 antihypertensive medicines in NRDL, there were sound evidence supports for the effectiveness of 56 kinds of medicines. Specifically, twenty-six of them were supported by international evidence-based guidelines, twenty were supported by systematic review or meta-analysis and the other ten by RCT. However, for the rest 41 medicines, there is insufficient evidence for their effectiveness.

Conclusions: Some antihypertensive medicines in NRDL did not have sufficient evidence for their effectiveness. Further evaluation and revision were required. It is also recommended to standardize decision-making procedures for inclusion of medicines, set up high quality evidence database to timely provide sound evidence, and so on.

Keywords: Evidence support, National Reimbursement Drug List (NRDL), Antihypertensive medicines, Urban employee basic medical insurance (UEBMI), China

\footnotetext{
* Correspondence: yychen@shmu.edu.cn

${ }^{5}$ Key Lab of Health Technology Assessment (Ministry of Health), Collaborative

Innovation Center of Social Risks Governance in Health, School of Public

Health, Fudan University, 446 Zhaojiabang Road Building 2 Room 1001,

Xuhui District, Shanghai 200032, China

Full list of author information is available at the end of the article
}

(c) The Author(s). 2019 Open Access This article is distributed under the terms of the Creative Commons Attribution 4.0 International License (http://creativecommons.org/licenses/by/4.0/), which permits unrestricted use, distribution, and reproduction in any medium, provided you give appropriate credit to the original author(s) and the source, provide a link to the Creative Commons license, and indicate if changes were made. The Creative Commons Public Domain Dedication waiver (http://creativecommons.org/publicdomain/zero/1.0/) applies to the data made available in this article, unless otherwise stated. 


\section{Background}

Evidence-based decision on drug list or formulary has been applied worldwide [1]. After proposing the recommendation that using evidence-based principles in the selection of essential medicines, World Health Organization (WHO) introduced the methods of Grading of Recommendations Assessment, Development and Evaluation (GRADE) into the document "WHO Handbook for Guideline Development- 2003”, which comprehensively take research design, quality and grade of evidences into consideration [2]. In 2009, WHO explicitly recommended to apply GRADE in evidence-based evaluation and selection of essential medicines, as well as submit the evidence of medicine efficacy and safety in the form of GRADE tables [2, 3].

In China, National Reimbursement Drug List (NRDL) of Urban Employee Basic Medical Insurance (UEBMI) is an important guidance list for production, supply, use, supervision, and management of included medicines. The medicines in this list are categorized into Class A and Class B. The medicines in Class A are often widely used in clinical treatment and have good curative effect, while the medicines in Class B alternatively using in clinical practice also have well treatment efficacy, but higher price [4]. The NRDL of UEBMI was firstly issued by Ministry of Labor and Social Security (MLSS) in 2000. The list was adjusted approximately every five years to make sure the included medicines are safe, effective, economic, and fulfilling basic clinical needs. The provincial bureaus have no right in adjusting medicines in Class $\mathrm{A}$, but they have the right to adjust not more than $15 \%$ of the medicines in Class B in accordance to local socio-economic level, health demand and medication habits [5].

Although the importance of scientific evidence was emphasized, which has also promoted the renewal and improvement in selection of medicines into the NRDL of UEBMI to some extent, the further improvement of the evidence-based evaluation system and working mechanism are still in urgent need for China [6, 7]. For many years, the list of alternative medicines to be included in NRDL of UEBMI was often formulated on the basis of national essential drug list and the need of certain medical services. To determine the medicines to be included in the updated NRDL, some experts were randomly selected from the MLSS expert database to vote. The medicines receiving more experts' support are likely to be included [8]. However, this decision-making procedure relies too much on expert opinion, which are mainly based on subjective experience rather than objective evidence. It greatly increases the risk of including inappropriate medicines in the NRDL, which may directly lead to inefficient use of health insurance funds and great adversity to public health.
For the wide coverage of UEBMI medicines, it is of great importance to use evidence-based methods to ensure their safety, efficacy, and cost-effectiveness. In consideration of their public health significance, this study takes the antihypertensive medicines as example to examine the situation of evidence support for the effectiveness of the medicines in the NRDL of UEBMI. The findings can provide recommendations for further revision of NRDL and the improvement of evidence-based decision-making.

\section{Methods}

To objectively judge whether the effectiveness of certain medicine was supported by sound evidence, we set a strategy by reviewing international evidence-based medicine lists in combination with published research paper of high evidence grade.

\section{Selection criteria of international evidence-based medicine lists}

Scientific evidence support and representative are two prerequisites for the selection of international evidence-based clinical guidelines or medicine lists as reference criteria. Representativeness implies the generality to a certain extent, while the scientific evidence support means the development of certain guidelines or drug lists following a formal and scientific procedure, such as searching and screening available high-level evidence, extracting, synthesizing and assessing data by rigorous methods, and so on.

Comprehensively considering the two elements mentioned above, three international evidence-based clinical guidelines or medicine list were selected: the WHO Essential Medicine List of 2017 version (EML 2017) [9], the Eighth Report of the Joint National Committee on Prevention, Detection, Evaluation and Treatment of high blood pressure (JNC 8) [10], and the Clinical Management of Primary Hypertension in Adults (NICE 2011) [11].

\section{Literature search strategy}

For each drug in the NRDL, a literature search was performed using the following databases: Pubmed, Medline, Web of science, China National Knowledge Infrastructure (CNKI), Wangfang Data, Chinese Bio-Medicine (CBM) database. The search started with broad search terms, such as the genetic name or commonly used brand name of certain medicine included in the current NRDL of UEBMI to treat hypertension, then narrowing down to the field of effectiveness with the time span between Jan 1st, 1995 and March 31st, 2017. 


\section{Literature inclusion criteria}

Clinical studies focusing on the certain medicines' effectiveness of the anti-hypertension with the research design as meta-analysis, systematic review, or randomized controlled trials between Jan 1st, 1995 and March 31st, 2017.

\section{Literature exclusion criteria}

(1) Studies mainly focusing on economic or cost analysis

(2) Studies that were not designed as meta-analysis, systematic review, or randomized controlled trials.

(3) Meta-analyses or systematic reviews that were not the latest ones on the effectiveness of certain medicines.

(4) Clinical studies not published in English or Chinese.

\section{Literature quality assessment}

The literature quality of the searched articles were subsequently reviewed and scored by two independent reviewers under the guidance of the scales or checklists mentioned below. If there were differences in the scores between the two reviewers, they were further discussed for consensus purpose.

\section{Quality assessment for systematic review and meta- analysis}

The reporting quality of systematic review and metaanalysis were assessed by using Preferred Reporting Items for Systematic Reviews and Meta-Analyses (PRISMA) statement. The checklist has 27 items about the literature integrity, such as objection in the introduction section, data collection process in the methods section, summary of evidence in the discussion section and so on [12]. If certain item was fully reported, partly reported, not reported at all, it will get 1 point, 0.5 point and 0 point, respectively. Therefore, the total score ranges from 0 to 27 points. Usually, systematic review or meta-analysis with PRISMA scoring more than 21 points can be considered fairly acceptable in literature integrity.

Meanwhile, the methodological quality of systematic review and meta-analysis were assessed by applying $\mathrm{A}$ Measurement Tool for the 'Assessment of multiple systematic reviews' (AMSTAR). The scale contains 11 items, such as "Was an "a priori" design provided?", "Was a list of studies (included and excluded) provided?", and so on. If the answer is "Yes", "Partly" and "No", it will get 1 point, 0.5 point and 0 point, respectively. Therefore, the total score ranges from 0 to 11 points [13]. By convention, the methodological quality of systematic review and meta-analysis with AMSTAR scoring more than 7 points can be considered acceptable.

\section{Quality assessment for RCT}

The methodological qualities of the included RCT were assessed by Jadad scale, which contains three items, such as "Randomization", "Double blinding" and "Withdrawals and dropouts" [14]. For the first 2 items, if certain item was "described and appropriate", "only described", "not mentioned", it will get 2 points, 1 point and 0 point, respectively. For the last item "Withdrawals and dropouts", if it was described, it will get 1 point, or else, 0 point. Therefore, the full mark of the adjusted Jadad scale is 5 points. Besides, the methodological quality of RCT with Jadad score not less than 3 points can be considered acceptable.

\section{Analysis strategy}

There are three steps as follows to assess the evidence for the effectiveness of anti-hypertensive medicines included on the NRDL.

Step one, comparison among the drug lists. If certain medicine included in NRDL of UEBMI was also included in at least two international evidence-based clinical guidelines or medicine lists mentioned above, it can be perceived that the effectiveness of this medicine was supported by sound evidence. Since the development of these evidence-based medicine lists following a scientific procedure which includes evidence searching and implementation from academic literature, these medicines supported by the evidence-based medicine lists will be not referred to published research paper for further assessment (No need to next step). However, if certain medicine included in NRDL of UEBMI was only included in one or none of these three lists, for considering many guidelines do not purport to provide an exhaustive list of all effective medicines, it will be referred to some published research paper with high-level evidence grade for further assessment.

Step two, collect evidence from systematic review or meta-analysis. For the medicines only included in one or none of these three international evidence-based lists, the systematic review or meta-analysis on their antihypertensive effectiveness will be reviewed. If certain medicine was confirmed effective in treating hypertension with low incidence of adverse events in meta-analysis or systematic review with acceptable literature quality, the effectiveness of this medicine can be also considered as having sound evidence support. Since systematic reviews or meta-analyses are the literature with highest evidence grade for its comprehensively combining the results of previous RCT, these medicines supported by meta-analysis or systematic review will be not referred to published RCT for further assessment (No need to next step). However, for the rest medicines having evidence support from neither international evidence-based medicine lists nor meta-analyses 
/ systematic reviews, they will be referred to published RCT for further assessment.

Step three, collect evidence from RCT. For the rest medicines mentioned above, RCTs on their effectiveness in treating hypertension will be reviewed. If certain medicine was reported by RCT with acceptable methodological quality as an effective antihypertensive medication with few adverse events, the effectiveness of this medicine can also be considered as having sound evidence support. Or else, the effectiveness of certain medicine in treating hypertension will be considered as having insufficient evidence.

\section{Statistical analysis}

The times of certain medicine included in the three-international evidence-based clinical guidelines on hypertension were calculated. For the medicines only included in one guideline or not included in any one of these three guidelines, the numbers of the kinds of medicines supported by published systematic review, meta-analysis and RCT were calculated respectively.

\section{Results}

\section{General information}

There are 97 antihypertensive medicines included in NRDL of UEBMI, which were divided into Class A and Class B. There are 26 medicines in Class A (26.8\%) and 71 included in Class B (73.2\%). Based on the mechanism of action, the 97 antihypertensive medicines can be divided into seven categories, such as calcium antagonist, $\beta$-blockers, diuretics, angiotensin converting enzyme inhibitors (ACEIs), Angiotensin II receptor antagonist, vasodilators and others, respectively accounting for 24.7, $14.4,11.4,15.5,12.4,7.2$ and $14.4 \%$ of all the 97 kinds of medicines.

\section{Evidence from the three international evidence-based clinical guidelines}

Twenty-six of the 97 surveyed medicines were included in all or two of the three international evidence-based clinical guidelines, which accounted for $26.8 \%$. The numbers of the kinds of medicines included in one of the three clinical guidelines were 16, accounting for $16.5 \%$ of the total. Additionally, fifty-five kinds of medicines (approximately 60\%) were included in neither of the guidelines (Shown in Table 1).

\section{Evidence from published literature with high evidence grade}

For the 71 kinds of medicines included in one or none of the three international evidence-based clinical guidelines, twenty-six of them had been evaluated in systematic review or meta-analysis, while 12 of them assessed in RCT. However, the other 33 medicines had not been reported in published literature with high evidence grade as mentioned above.

\section{Evidence from systematic review and meta-analysis}

For all the 25 systematic reviews and meta-analyses initially included, their PRISMA scores were more than 21 points, which infers that the literature integrity can be considered fairly acceptable (See column 5, Table 2). With respect to the methodological quality assessed by the AMSTAR scale, the AMSTAR scores were between 7 and 11 points for all the 25 included systematic reviews and meta-analyses, which can be considered that their methodological quality were good (Shown in column 4, Table 2). And the completion of each items in AMSTAR Scale are shown in Additional file 1: Table S1.

The results published in these systematic review and meta-analysis indicated that fosinpril [15], perindopril [16], carvedilol [17], Doxazoxin [18], Urapidil (injection) [19], Cilnidipine [20], Sodium nitroprusside [21], Lacidipine [22], Nicardipine (injection) [23], Lercanidipine [24], Arotinolol [25], Benazepril [26], Enopril folic acid [27], Irbesartan hydrochlorothiazide [28], Valsartan hydrochlorothiazide [29], Levamlodipine besylate [30], Nimodipine (oral dosage) [31], Compound reserpine ammonia benzene pteridine [32], Labetalol [33], and Compound reserpine [34] were fairly effective in treating hypertension with low incidence rate of adverse reactions. In other words, the effectiveness of regarding medicines were supported by these systematic review and meta-analysis. However, for Esmolol [35], Nitrendipine [36], Felodipine [37], Felodipine II [37], Hydralazine [38] and Reserpine [39], their effectiveness in treating hypertension were not confirmed in regarding systematic review or meta-analysis. For instance, the reported results pointed out that the data on efficacy and safety of Hydralazine was based on before and after studies, not RCTs. The efficacy of Hydralazine in treating hypertension remains uncertain [38]. (See Table 2).

\section{Evidence from RCT}

Fourteen RCTs were initially included in this study and their methodological qualities were assessed by the adjusted Jadad scale. Eleven of them had the Jadad score not less than 3 points, while the other three had the scores between 0 and 2 points. (See Additional file 2: Table S2).

Although the safety and effectiveness of Amlodipine benapril [40], Metoprolol (injection) [41], were reported in some RCTs, the Jadad score of these RCTs were all between 0 and 2 points, which were considered as unacceptable methodological quality. Thus, the conclusions of these studies should be cautious to use, which implies uncertainty of the effectiveness of these medicines. For Propranolol (injection), its effectiveness of 
Table 1 Evidence for the effectiveness of anti-hypertensive drugs in NRDL of UEBMI_—From the international evidence-based drug list

Dosage form $\quad$ JNC 8 NICE 2011 WHO EML 2017 Frequency of

Calcium antagonist

Class A

Amlodipine
Nimodipine
Nitrendipine
Nifedipine
Diltiazem
Verapamil
Verapamil

oral release dosage form

oral release dosage form

oral release dosage form

oral release dosage form

oral release dosage form

oral release dosage form

injection inclusion

Class B

L - nmda amlodipine

Amlodipine atorvastatin calcium

oral release dosage form

oral release dosage form

Benidipine

Felodipine

Felodipine II

Lacidipine

Lercanidipine

Nicardipine

Nicardipine

oral release dosage form

oral release dosage form, controlled release dosage form

controlled release dosage form

oral release dosage form

oral release dosage form

oral release dosage form, controlled release dosage form

injection

Nimodipine

Nitrendipine and Atenolol

injection

Cilnidipine

Nifedipine $(I, I,|I|)$

Levamlodipine besylate tablets

Diltiazem

Diltiazem (II)

Verapamil

oral release dosage form

oral release dosage form

controlled release dosage form

oral release dosage form

injection

controlled release dosage form

controlled release dosage form

$\begin{array}{lccc}\sqrt{ } & \sqrt{ } & \sqrt{ } & 3 \\ - & - & - & 0 \\ - & - & - & 0 \\ - & \sqrt{ } & \sqrt{ } & 2 \\ - & \sqrt{ } & \sqrt{ } & 2 \\ - & \sqrt{ } & \sqrt{ } & 2 \\ - & - & \sqrt{ } & 1\end{array}$

$\beta$ blockers

Class A

Propranolol

Atenolol

Bisoprolol

Metoprolol

Metoprolol

Class B

Propranolol

Propranolol

Sotalol

Sotalol

Esmolol

Metoprolol oral release dosage form

oral release dosage form

oral release dosage form

oral release dosage form

injection

Sustained or controlled release dosage form injection

oral release dosage form

injection

injection

controlled release dosage form

$\begin{array}{llll}- & - & - & 0 \\ - & - & - & 0 \\ - & - & - & 0 \\ - & \sqrt{ } & - & 1 \\ - & \sqrt{ } & - & 1 \\ - & \sqrt{ } & - & 1 \\ - & \sqrt{ } & - & 1 \\ - & - & - & 0 \\ & & & \\ - & - & - & 0 \\ - & - & - & 0 \\ - & - & - & 0 \\ - & - & - & 0 \\ - & \sqrt{ } & \sqrt{ } & 2 \\ - & \sqrt{ } & - & 1 \\ - & - & - & 0 \\ - & \sqrt{ } & \sqrt{ } & 2 \\ - & \sqrt{ } & \sqrt{ } & 2\end{array}$

$\begin{array}{cccc}- & \sqrt{ } & \sqrt{ } & 2 \\ \sqrt{ } & \sqrt{ } & - & 2 \\ - & \sqrt{ } & \sqrt{ } & 2 \\ \sqrt{ } & \sqrt{ } & - & 2 \\ - & - & - & 0\end{array}$

$\begin{array}{llll}- & \sqrt{ } & \sqrt{ } & 2 \\ - & - & - & 0 \\ - & \sqrt{ } & - & 1 \\ - & - & - & 0 \\ - & - & - & 0 \\ \sqrt{ } & \sqrt{ } & - & 2\end{array}$


Table 1 Evidence for the effectiveness of anti-hypertensive drugs in NRDL of UEBMI_—From the international evidence-based drug list (Continued)

\begin{tabular}{|c|c|c|c|c|c|}
\hline & Dosage form & JNC 8 & NICE 2011 & WHO EML 2017 & $\begin{array}{l}\text { Frequency of } \\
\text { inclusion }\end{array}$ \\
\hline Arotinolol & oral release dosage form & - & - & - & 0 \\
\hline Carvedilol & oral release dosage form & - & - & - & 0 \\
\hline Labetalol & oral release dosage form & - & - & - & 0 \\
\hline \multicolumn{6}{|l|}{ diuretics } \\
\hline \multicolumn{6}{|l|}{ Class A } \\
\hline Hydrochlorthiazide & oral release dosage form & $\sqrt{ }$ & $\sqrt{ }$ & $\sqrt{ }$ & 3 \\
\hline Indapamide & $\begin{array}{l}\text { oral release dosage form, controlled release } \\
\text { dosage form }\end{array}$ & $\sqrt{ }$ & $\sqrt{ }$ & - & 2 \\
\hline Furosemide & oral release dosage form & - & - & $\sqrt{ }$ & 1 \\
\hline Furosemide & injection & - & - & $\sqrt{ }$ & 1 \\
\hline Triamterene & oral release dosage form & - & - & - & 0 \\
\hline Spironolactone & oral release dosage form & - & $\sqrt{ }$ & $\sqrt{ }$ & 2 \\
\hline \multicolumn{6}{|l|}{ Class B } \\
\hline Bumetanide & oral release dosage form & - & - & - & 0 \\
\hline Bumetanide & injection & - & - & - & 0 \\
\hline Torasemide & oral release dosage form & - & - & - & 0 \\
\hline Torasemide & injection & - & - & - & 0 \\
\hline Amiloride & oral release dosage form & - & $\sqrt{ }$ & $\sqrt{ }$ & 2 \\
\hline \multicolumn{6}{|l|}{ ACEls } \\
\hline \multicolumn{6}{|l|}{ Class A } \\
\hline Captopril & oral release dosage form & $\sqrt{ }$ & $\sqrt{ }$ & - & 2 \\
\hline Enalapril & oral release dosage form & $\sqrt{ }$ & $\sqrt{ }$ & $\sqrt{ }$ & 3 \\
\hline \multicolumn{6}{|l|}{ Class B } \\
\hline Benazepril & oral release dosage form & - & - & - & 0 \\
\hline Fosinopril & oral release dosage form & - & - & - & 0 \\
\hline Lisinopril & oral release dosage form & $\sqrt{ }$ & $\sqrt{ }$ & - & 2 \\
\hline Ramipril & oral release dosage form & - & $\sqrt{ }$ & - & 1 \\
\hline Imidapril & oral release dosage form & - & - & - & 0 \\
\hline Perindopril & oral release dosage form & - & $\sqrt{ }$ & - & 1 \\
\hline Cilazapril & oral release dosage form & - & - & - & 0 \\
\hline Amlodipine benapril I (II) & oral release dosage form & - & - & - & 0 \\
\hline Benapril hydrochlorothiazide & oral release dosage form & - & - & - & 0 \\
\hline compound captopril & oral release dosage form & - & - & - & 0 \\
\hline Lynopli hydrochlorothiazide & oral release dosage form & - & - & - & 0 \\
\hline Enalpril hydrochlorothiazide & oral release dosage form & - & - & - & 0 \\
\hline Enopril folic acid & oral release dosage form & - & - & - & 0 \\
\hline \multicolumn{6}{|l|}{ Angiotensin II receptor antagonist } \\
\hline \multicolumn{6}{|l|}{ Class B } \\
\hline Olmesartan Medoxomil & oral release dosage form & - & - & - & 0 \\
\hline Irbesartan & $\begin{array}{l}\text { oral release dosage form, controlled release } \\
\text { dosage form }\end{array}$ & $\sqrt{ }$ & $\sqrt{ }$ & - & 2 \\
\hline Candesartan & oral release dosage form & $\sqrt{ }$ & $\sqrt{ }$ & - & 2 \\
\hline Losartan & oral release dosage form & $\sqrt{ }$ & $\sqrt{ }$ & $\sqrt{ }$ & 3 \\
\hline
\end{tabular}


Table 1 Evidence for the effectiveness of anti-hypertensive drugs in NRDL of UEBMI_—From the international evidence-based drug list (Continued)

\begin{tabular}{|c|c|c|c|c|c|}
\hline & Dosage form & JNC 8 & NICE 2011 & WHO EML 2017 & $\begin{array}{l}\text { Frequency of } \\
\text { inclusion }\end{array}$ \\
\hline Telmisartan & oral release dosage form & $\sqrt{ }$ & $\sqrt{ }$ & - & 2 \\
\hline Valsartan & oral release dosage form & $\sqrt{ }$ & $\sqrt{ }$ & - & 2 \\
\hline Omethane hydrochlorothiazide & oral release dosage form & - & - & - & 0 \\
\hline irbesartan hydrochlorothiazide & oral release dosage form & - & - & - & 0 \\
\hline chlorothiazide & oral release dosage form & - & - & - & 0 \\
\hline Timisaltan hydrochlorothiazide & oral release dosage form & - & - & - & 0 \\
\hline Valsartan chlordipine I (II) & oral release dosage form & - & - & - & 0 \\
\hline Valsartan hydrochlorothiazide & oral release dosage form & - & - & - & 0 \\
\hline \multicolumn{6}{|l|}{ vasodilators } \\
\hline \multicolumn{6}{|l|}{ Class A } \\
\hline Phentolamine & injection & - & - & - & 0 \\
\hline Sodium nitroprusside & injection & - & - & $\sqrt{ }$ & 1 \\
\hline \multicolumn{6}{|l|}{ Class B } \\
\hline Phenoxybenzamine & oral release dosage form & - & - & - & 0 \\
\hline Phenoxybenzamine & injection & - & - & - & 0 \\
\hline Hydralazine & oral release dosage form & - & - & $\sqrt{ }$ & 1 \\
\hline Hydralazine & injection & - & - & $\sqrt{ }$ & 1 \\
\hline Minoxidil & oral release dosage form & - & - & - & 0 \\
\hline \multicolumn{6}{|l|}{ Others } \\
\hline \multicolumn{6}{|l|}{ Class A } \\
\hline Reserpine & injection & - & - & - & 0 \\
\hline Prazosin & oral release dosage form & - & $\sqrt{ }$ & - & 1 \\
\hline Compound reserpine & oral release dosage form & - & - & - & 0 \\
\hline $\begin{array}{l}\text { Compound reserpine ammonia benzene } \\
\text { pteridine }\end{array}$ & oral release dosage form & - & - & - & 0 \\
\hline \multicolumn{6}{|l|}{ Class B } \\
\hline Dibazole & oral release dosage form & - & - & - & 0 \\
\hline Methyldopa & oral release dosage form & - & $\sqrt{ }$ & $\sqrt{ }$ & 2 \\
\hline Clonidine & oral release dosage form & - & - & - & 0 \\
\hline Clonidine & Patch & - & - & - & 0 \\
\hline Reserpine & oral release dosage form & - & - & - & 0 \\
\hline Ligustrazine & Injection & - & - & - & 0 \\
\hline Doxazosin & $\begin{array}{l}\text { oral release dosage form, controlled release } \\
\text { dosage form }\end{array}$ & - & $\sqrt{ }$ & - & 1 \\
\hline Naftopidil & oral release dosage form & - & - & - & 0 \\
\hline Urapidil & $\begin{array}{l}\text { oral release dosage form, controlled release } \\
\text { dosage form }\end{array}$ & - & - & - & 0 \\
\hline Urapidil & injection & - & - & - & 0 \\
\hline
\end{tabular}

treating hypertension was also confirmed by one RCT published in 2007 [42], though another one on its effectiveness published in 2001 has unacceptable methodological quality [43]. Additionally, as reported by the other 10 RCTs with Jadad score not less than 3 points. Furosemide (injection) [44], Torasemide (injection)
[45], Naftopidil [46], Olmesartan Medoxomil [47], Ramipril [48, 49], Imidapril [50], Diltiazem (injection) [51], Enalpril hydrochlorothiazide [52] and Cilazapril [53] were fairly effective in treating hypertension with few adverse events. Therefore, it can be considered that the effectiveness of these five medicines were supported 
Table 2 Evidence for effectiveness of anti-hypertensive medicines in NRDL of UEBMI__From systematic review and meta-analysis

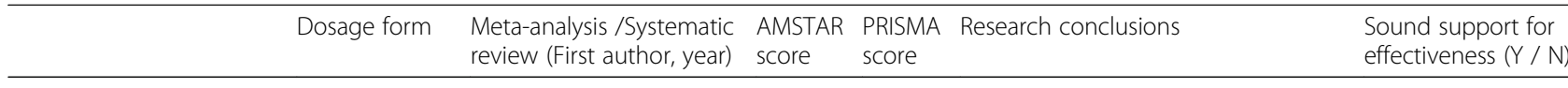

Calcium antagonist

Class A

Nimodipine

Nitrendipine

oral release

dosage form

Du X, 2014 [32]

Class B

Levamlodipine besylate

Felodipine

Felodipine ||

Lercanidipine

oral release dosage form

Ran Y, 2015 [20]

oral release

dosage form

controlled

release dosage

form

controlled

release dosage

form

Nicardipine

injection

Jiang C, 2013 [19]

Lacidipine

oral release

dosage form

Cilnidipine

oral release

dosage form

Li S, 2012 [16]

Hua Q, 2014 [18]

9.5

23

B-blockers

Class B

$$
\text { Arotinolol }
$$

oral release

dosage form

Du B, 2009 [21]

8.5

21.5

oral release

Labetalol dosage form
For patients with $\mathrm{HICH}$, nimodipine $\quad \mathrm{Y}$

combined with edaravone has significant clinical efficacy in the treatment of hypertensive cerebral hemorrhage, and can improve the functional rehabilitation of the nerves and effectively reduce the volumes of intracranial hematoma and peripheral edema.

In lowering blood pressure,

Amlodipine is better than

Nitrendipine in the cost and effect.

Levamlodipine is more effective and safer than amlodipine in treatment of mild to moderate hypertension than, which is thus worthy of clinical application.

In the treatment of Chinese patients N with hypertension, amlodipine both in reducing systolic or diastolic pressure were better than felodipine, no statistical significance on both heart rate and adverse drug reaction differences.

In the treatment of Chinese patients N with hypertension, amlodipine both in reducing systolic or diastolic pressure were better than felodipine, no statistical significance on both heart rate and adverse drug reaction differences.

Based on the current evidence, the $Y$ safety and compliance of lercanidipine is better than amlodipine in the treatment of mild to moderate hypertension.

Nicardipine was safe and effective in $Y$ the treatment of hypertensive emergency.

Lacidipine has been similar to amlodipine in the treatment of essential hypertension with less adverse drug reaction.

Cilnidipine has the similar efficacy and safety compared with control group in treating essential hypertension.

In treating essential hypertension, $\quad Y$ there is no significant difference in efficacy and safety between Arotinolol and control group, such as Cilnidipine, felodipine and Imidapril.

Labetalol is a reasonable choice for $Y$ treatment of severe or non-severe 
Table 2 Evidence for effectiveness of anti-hypertensive medicines in NRDL of UEBMI_—From systematic review and meta-analysis (Continued)

\begin{tabular}{|c|c|c|c|c|c|c|}
\hline & Dosage form & $\begin{array}{l}\text { Meta-analysis /Systematic } \\
\text { review (First author, year) }\end{array}$ & $\begin{array}{l}\text { AMSTAR } \\
\text { score }\end{array}$ & $\begin{array}{l}\text { PRISMA } \\
\text { score }\end{array}$ & Research conclusions & $\begin{array}{l}\text { Sound support for } \\
\text { effectiveness }(Y / N)\end{array}$ \\
\hline & & & & & hypertension in pregnancy & \\
\hline Carvedilol & $\begin{array}{l}\text { oral release } \\
\text { dosage form }\end{array}$ & Chen S, 2015 [13] & 9 & 24 & $\begin{array}{l}\text { Carvedilol has a greater portal } \\
\text { hypertensive effect than propranolol. } \\
\text { Further comparative trials of the two } \\
\text { drugs are required to identify the } \\
\text { effect of MAP reduction. }\end{array}$ & Y \\
\hline Esmolol & injection & $\begin{array}{l}\text { Garnockjones KP, } 2012 \\
\text { [31] }\end{array}$ & 10 & 25 & $\begin{array}{l}\text { Definitive conclusions on the efficacy } \\
\text { of esmolol are difficult to reach, as } \\
\text { most trials investigating esmolol have } \\
\text { limitations such as small patient } \\
\text { populations, and few studies } \\
\text { investigate the same parameters. }\end{array}$ & N \\
\hline
\end{tabular}

ACEIS

Class B

\begin{tabular}{|c|c|c|c|c|c|}
\hline Perindopril & $\begin{array}{l}\text { oral release } \\
\text { dosage form }\end{array}$ & Gasowski J, 2010 [12] & 8.5 & 24 & $\begin{array}{l}\text { Perindopril is an effective } \\
\text { antihypertensive medication. Seems } \\
\text { not to be adversely affected by the } \\
\text { clinical profile of the patient. }\end{array}$ \\
\hline Benazepril & $\begin{array}{l}\text { oral release } \\
\text { dosage form }\end{array}$ & Zhao S, 2015 [22] & 7.5 & 22 & $\begin{array}{l}\text { Benazepril can more effectively } \\
\text { lower the blood pressure of patients } \\
\text { with primary hypertension than } \\
\text { captopril. }\end{array}$ \\
\hline Fosinopril & $\begin{array}{l}\text { oral release } \\
\text { dosage form }\end{array}$ & Zeng X, 2014 [11] & 8.5 & 23.5 & $\begin{array}{l}\text { The curative effect of fosinopril is } \\
\text { almost the same as calcium } \\
\text { antagonists in the treatment of mild } \\
\text { to moderate essential hypertension, } \\
\text { but superior to other types of } \\
\text { antihypertensive drugs and shows } \\
\text { good safety. }\end{array}$ \\
\hline Enopril folic acid & $\begin{array}{l}\text { oral release } \\
\text { dosage form }\end{array}$ & Zhang Y, 2015 [23] & 7.5 & 22 & $\begin{array}{l}\text { Enopril folic acid showed better } \\
\text { efficacy in lowering blood pressure } \\
\text { and preventing cardiovascular } \\
\text { accident than Enopril. }\end{array}$ \\
\hline
\end{tabular}

Vasodilators

Class A

Sodium

nitroprusside

injection

Dong W, 2012 [17]

Sodium nitroprusside is effective for $\quad Y$ the treatment of hypertensive emergency, while the ADRs are acceptable.

Class B

Hydralazine

oral release

Kandler M R, 2010 [34]

11

27

Hydralazine may reduce blood

pressure when compared to placebo in patients with primary hypertension, however this data is based on before and after studies, not RCTs. Furthermore, its effect on clinical outcomes remains uncertain.

\section{Angiotensin II receptor antagonist}

Class B

$\begin{array}{ll}\text { Irbesartan } & \text { oral release } \\ \text { /Hydrochlorothiazide } & \text { dosage form }\end{array}$

Wu H, 2011 [24]

/Hydrochlorothiazide dosage form

Valsartan

oral release

Jin J, 2013 [25]

8
21

Irbesartan/hydrochlorothiazide combination therapy is more effective than control group in treating essential hypertension and there is no significant difference in safety.

In the treatment of primary dosage form 
Table 2 Evidence for effectiveness of anti-hypertensive medicines in NRDL of UEBMI_—From systematic review and meta-analysis (Continued)

\begin{tabular}{|c|c|c|c|c|c|c|}
\hline & Dosage form & $\begin{array}{l}\text { Meta-analysis /Systematic } \\
\text { review (First author, year) }\end{array}$ & $\begin{array}{l}\text { AMSTAR } \\
\text { score }\end{array}$ & $\begin{array}{l}\text { PRISMA } \\
\text { score }\end{array}$ & Research conclusions & $\begin{array}{l}\text { Sound support for } \\
\text { effectiveness }(Y / N)\end{array}$ \\
\hline & & & & & $\begin{array}{l}\text { Hydrochlorothiazide combination } \\
\text { has better anti-hypertension efficacy } \\
\text { with less adverse drug reaction. }\end{array}$ & \\
\hline \multicolumn{7}{|l|}{ Others } \\
\hline \multicolumn{7}{|l|}{ Class A } \\
\hline $\begin{array}{l}\text { Compound } \\
\text { reserpine }\end{array}$ & $\begin{array}{l}\text { oral release } \\
\text { dosage form }\end{array}$ & Hu L, 2012 [30] & 9 & 25.5 & $\begin{array}{l}\text { Based on current research evidence, } \\
\text { Compound reserpine tablets are } \\
\text { safe and effective. }\end{array}$ & Y \\
\hline $\begin{array}{l}\text { Compound } \\
\text { reserpine ammonia } \\
\text { benzene pteridine }\end{array}$ & $\begin{array}{l}\text { oral release } \\
\text { dosage form }\end{array}$ & Wu Y, 2009 [28] & 9 & 23 & $\begin{array}{l}\text { Compound reserpine ammonia } \\
\text { benzene pteridine appears to have } \\
\text { the same effect as other anti- } \\
\text { hypertensive drugs without having } \\
\text { more adverse events. }\end{array}$ & Y \\
\hline \multicolumn{7}{|l|}{ Class B } \\
\hline Doxazosin & $\begin{array}{l}\text { oral release } \\
\text { dosage form }\end{array}$ & Ke Z, 2015 [14] & 8.5 & 24 & $\begin{array}{l}\text { Doxazosin has affirmed effect on } \\
\text { mild to moderate essential } \\
\text { hypertension, with little side effect, } \\
\text { good patient tolerance, especially } \\
\text { for hypertension patients with } \\
\text { benign prostatic hyperplasia. }\end{array}$ & Y \\
\hline Urapidil & injection & Zhou X, 2016 [15] & 7.5 & 21.5 & $\begin{array}{l}\text { Urapidil and conventional } \\
\text { vasedilator like Nitroglycerin are } \\
\text { effective to heart failure with } \\
\text { hypertension. For lowering blood } \\
\text { pressure, their efficacy are equal, } \\
\text { but for reducing patients BNP and } \\
\text { heart rate, Urapidil is better than } \\
\text { the other. }\end{array}$ & Y \\
\hline Reserpine & $\begin{array}{l}\text { oral release } \\
\text { dosage form }\end{array}$ & Shamon S D, 2009 [35] & 9 & 25 & $\begin{array}{l}\text { Reserpine is effective in reducing } \\
\text { SBP roughly to the same degree as } \\
\text { other first-line antihypertensive } \\
\text { drugs. However, we could not make } \\
\text { definite conclusions regarding the } \\
\text { dose-response pattern because of } \\
\text { the small number of included trials. } \\
\text { More RCTs are needed to assess the } \\
\text { effects of reserpine on blood } \\
\text { pressure and to determine the } \\
\text { dose-related safety profile before } \\
\text { the role of this drug in the } \\
\text { treatment of primary hypertension } \\
\text { can be established. }\end{array}$ & N \\
\hline
\end{tabular}

by RCTs with acceptable methodological quality. (See Table 3).

\section{Overall situation of evidence support}

Taking the evidence from three international evidence-based clinical guidelines on hypertension, systematic review/meta-analysis, and RCT, as well as the methodological quality of the literature comprehensively into account, the effectiveness of 56 kinds of medicines were supported by fairly sound evidence. To be specific, twenty-six of the medicines were supported by international evidence-based clinical guideline on hypertension, while twenty were supported by systematic review/ meta-analysis and the other ten supported by RCT.
However, for the other 41 kinds of medicines, two out of them were supported by RCTs with poor methodological quality, thirty-three of them did not gain support from the evidence with high grade, and six medicines were reported uncertainty of antihypertensive effectiveness in meta-analysis or systematic review. (See Table 4).

With regard to the antihypertensive medicines with different major functions, the evidence support status was also very different among them. The proportion of the medicines with confirmed effectiveness ranged from 14.3\% (for Vasodilators) to 73.3\% (for ACEIs). Regarding to the different management categories, approximately $70 \%$ of the antihypertensive medicines in Class A have sound evidence support for their effectiveness, while the 
Table 3 Evidence for effectiveness of anti-hypertensive medicines in NRDL of UEBMI_—From RCT

\begin{tabular}{|c|c|c|c|c|}
\hline & $\begin{array}{l}\text { Dosage } \\
\text { form }\end{array}$ & RCT (First author, year) & $\begin{array}{l}\text { JADAD } \\
\text { score }\end{array}$ & Research conclusions \\
\hline \multicolumn{5}{|l|}{ ACEls } \\
\hline \multicolumn{5}{|l|}{ Class B } \\
\hline $\begin{array}{l}\text { Amlodipine } \\
\text { benapril }\end{array}$ & $\begin{array}{l}\text { oral release } \\
\text { dosage form }\end{array}$ & Cao J, 2012 [36] & 2 & $\begin{array}{l}\text { Amlodipine benapril can achieve better } \\
\text { antihypertensive effect and reduce } \\
\text { adverse reactions, which is worthy of } \\
\text { clinical promotion. }\end{array}$ \\
\hline $\begin{array}{l}\text { Enalpril } \\
\text { hydrochlorothiazide }\end{array}$ & $\begin{array}{l}\text { oral release } \\
\text { dosage form }\end{array}$ & Wang X, 2006 [48] & 4 & $\begin{array}{l}\text { The efficacy of combination of enalapril } \\
\text { hydrochlorothiazide is better than single } \\
\text { use of enalapril in the treatment of mild } \\
\text { and moderate primary hypertension. } \\
\text { Combination of enalapril } \\
\text { hydrochlorothiazide has the same safety } \\
\text { and toleration as enalapril. }\end{array}$ \\
\hline Imidapril & $\begin{array}{l}\text { oral release } \\
\text { dosage form }\end{array}$ & Jiang X, 2005 [46] & 4 & $\begin{array}{l}\text { Imidapril and Benapril are both similarly } \\
\text { effective in the reduction of the } \\
\text { peripheral blood pressure and the central } \\
\text { blood pressure. }\end{array}$ \\
\hline \multirow[t]{2}{*}{ Ramipril } & $\begin{array}{l}\text { oral release } \\
\text { dosage form }\end{array}$ & Tao B, 2006 [44] & 4 & $\begin{array}{l}\text { The combination of irbesartan with } \\
\text { felodipine or ramipril showed synergist } \\
\text { antihypertensive effects. Moreover, the } \\
\text { combination of irbesartan with felodipine } \\
\text { was superior to combination of irbesartan } \\
\text { with ramipril. }\end{array}$ \\
\hline & & Rokoss M J, 2005 [45] & 5 & $\begin{array}{l}\text { Beneficial effects of ramipril are observed } \\
\text { in the treatment of hypertension and } \\
\text { congestive heart failure, prevention of } \\
\text { cardiovascular events in high-risk patients, } \\
\text { prevention of congestive heart failure, } \\
\text { diabetes and other vascular events }\end{array}$ \\
\hline Cilazapril & $\begin{array}{l}\text { oral release } \\
\text { dosage form }\end{array}$ & Schiffrin EL, 2008 [49] & 4 & $\begin{array}{l}\text { These results may indicate that treatment } \\
\text { with cilazapril and perhaps with other } \\
\text { angiotensin-l- converting enzyme inhibitors } \\
\text { as well may improve the clinical outcome } \\
\text { in hypertension by inducing a regression of } \\
\text { abnormal resistance vessel structure and } \\
\text { function. }\end{array}$ \\
\hline
\end{tabular}

$\beta$-blockers

Class A

Metoprolol injection Lu N, 2006 [37]

Metoprolol is effective and safe for patients with unstable angina pectoris. It may significantly lower the risk of refractory angina pectoris.

Class B

Propranolol_ injection Jiang X, 2001 [39]

Zuo W, 2007 [38]

Calcium antagonist

Class B

Diltiazem

injection
Sound support for effectiveness $(\mathrm{Y} / \mathrm{N})$

Y with cilazapril and perhaps with other function. 
Table 3 Evidence for effectiveness of anti-hypertensive medicines in NRDL of UEBMI__From RCT (Continued)

\begin{tabular}{|c|c|c|c|c|c|}
\hline & $\begin{array}{l}\text { Dosage } \\
\text { form }\end{array}$ & RCT (First author, year) & $\begin{array}{l}\text { JADAD } \\
\text { score }\end{array}$ & Research conclusions & $\begin{array}{l}\text { Sound support } \\
\text { for effectiveness } \\
(Y / N)\end{array}$ \\
\hline & & $2005[47]$ & & $\begin{array}{l}\text { and safe for patients with unstable angina } \\
\text { pectoris. It may significantly lower the risk } \\
\text { of refractory angina pectoris compared } \\
\text { with intravenous nitroglycerin. }\end{array}$ & \\
\hline \multicolumn{6}{|l|}{ Diuretics } \\
\hline \multicolumn{6}{|l|}{ Class A } \\
\hline Furosemide & injection & Huang G, 2008 [40] & 3 & $\begin{array}{l}\text { The use of } 125 \mathrm{ml} 20 \% \text { mannitol each time } \\
\text { plus } 20 \mathrm{mg} \text { furosemide is more reasonable } \\
\text { than other combinations. Meanwhile, semis } \\
\text { mannitol combined with moderate or large } \\
\text { dose of albumin has certain advantages too. }\end{array}$ & Y \\
\hline \multicolumn{6}{|l|}{ Class B } \\
\hline Torasemide & injection & Zheng W, 2008 [41] & 3 & $\begin{array}{l}\text { Torasemide injection is an effective and } \\
\text { safe drug for the treatment of congestive } \\
\text { heart failure with edema. }\end{array}$ & Y \\
\hline \multicolumn{6}{|c|}{ Angiotensin II receptor antagonist } \\
\hline \multicolumn{6}{|l|}{ Class B } \\
\hline $\begin{array}{l}\text { Olmesartan } \\
\text { Medoxomil }\end{array}$ & $\begin{array}{l}\text { oral release } \\
\text { dosage form }\end{array}$ & Liao Y, 2014 [43] & 3 & $\begin{array}{l}\text { Compared with enalapril, Olmesartan } \\
\text { Medoxomil has more significant } \\
\text { inhibition and reversal effects of left } \\
\text { ventricular remodeling in treatment of } \\
\text { the morning surge of hypertension. }\end{array}$ & Y \\
\hline \multicolumn{6}{|l|}{ Others } \\
\hline \multicolumn{6}{|l|}{ Class B } \\
\hline Naftopidil & $\begin{array}{l}\text { oral release } \\
\text { dosage form }\end{array}$ & Lu Q, 2000 [42] & 3 & $\begin{array}{l}\text { The efficacy of Naftopidil in controlling } \\
\text { the blood pressure is as efficient as that } \\
\text { of Terazosin and both drugs are well } \\
\text { tolerated by the patients. }\end{array}$ & Y \\
\hline
\end{tabular}

corresponding proportion is about $50 \%$ for the antihypertensive medicines in Class B. (See Table 4).

\section{Discussion}

This study applied a rapid evaluation strategy to determine the situation of evidence support for the effectiveness of medicines. It reveals that there is insufficient evidence of effectiveness for approximately $45 \%$ of the antihypertensive medicines included in the NRDL of UEBMI. The results with policy implication will provide a reference for the adjustment and improvement of the NRDL. Moreover, a series of factors leading to these results deserved further consideration.

Firstly, since the JNC8, NICE 2011, and EML 2017 are all evidence-based drug lists, the great differences between NRDL of UEBMI and three international evidence-based drug lists can not be ignored. To explore the underlying reasons for such discordance, the regional diversity may be the plausible. For population differences in response to antihypertensive treatments, the decision-makers may include other medicines in the NRDL to meet local needs, and subsequently lead to large differences between NRDL and international evidence-based drug list. However, whether the differences are reasonable still remains to be intensively determined.

To this point, it is important to fully examine published literature with high evidence grade, such as meta-analysis, systematic review, and RCT. In this study, $20.6 \%$ (20/97) of antihypertensive medicines were supported by meta-analysis or systematic review with regard to their effectiveness, while only $10.3 \%$ (10/97) of antihypertensive medicines were confirmed by RCT. Besides, it is worth noting that nearly $30 \%$ of the included RCTs had unacceptable quality, which implied that the methodological quality of RCT needs further improvement. Some shortcomings, such as inappropriate method in description of withdrawals and dropouts and failure in double blinding, had greatly deteriorated the authenticity and reliability of RCT. For the sake of further improving the methodological or report quality of systematic review, meta-analysis and RCT, it is recommended to provide researchers with methodological trainings on special issues, such as proper quality evaluation methods for meta-analysis, systematic review and RCT, appropriate method for applying randomization and blinding 


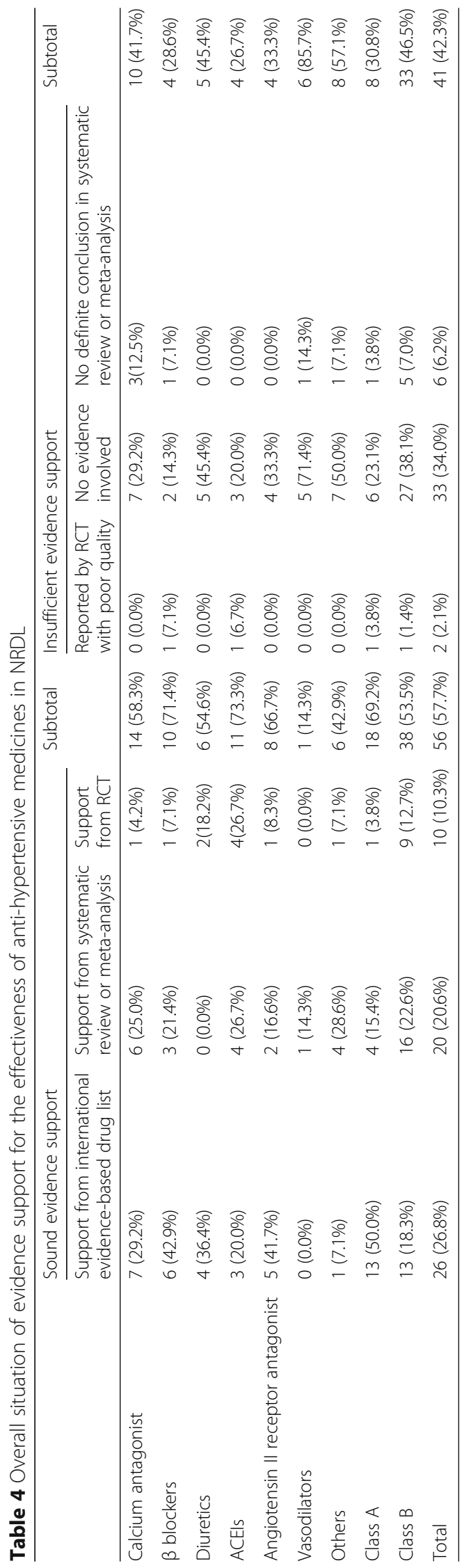


[54]. Additionally, for ensuring high quality of scientific evidence, strict monitoring on methodology as well as report quality of submitted manuscript before its publication is strongly recommended.

With regard to the overall situation of evidence support for the effectiveness, only a small proportion of the medicines among vasodilators were confirmed by sound evidence, which highlights the need of further adjustment for certain kinds of medicines. Additionally, in comparison with Class B, a larger proportion of antihypertensive medicines in Class A were confirmed by sound evidence, which demonstrates that more deliberate management had been implemented on essential and commonly used medicines.

However, it is still noteworthy that there is insufficient evidence for the effectiveness of almost $45 \%$ of the antihypertensive medicines in the NRDL of UEBMI. What is more, six of them were demonstrated by meta-analysis or systematic review as having no definitive conclusion on the effectiveness of treating hypertension. Although it can be more prudent to wait for more evidence, these medicines were still included in the NRDL. There are some reasons underlying this fact. One plausible reason may be the price issue even though it is not the focus of this paper. There is no denying that the drugs with less efficacies but lower prices also tended to be included in the insurance drug list for their potential great economic accessibility. Besides, the influence of the decision-making process of medicine inclusion in China also can not be ignored. It is an expert reviews system with hundreds or even thousands of experts involved to provide their opinions on the safety, clinical efficacy and economic cost of certain medicine. And the decision will be made to include or exclude certain medicine from the NRDL by comprehensively taking the experts' opinion into account [55]. Apparently, this decision-making process mainly depends on the expert opinion and seems more efficient than the process of "evidence collectionevidence evaluation-evidence application-decisionmaking". However, since it can hardly identify whether the expert opinions were based on "evidence" rather than "experience", its potential risk of decision-making error would be much higher. For the timeliness of decision-making and the practicality of medical practice, it will be more sensible to combine expert opinion with scientific evidence [56].

To promote scientific and efficient decision-making, some recommendations are also proposed as two folds: on the one hand, we should steadily improve the methodological quality of academic literature, such as meta-analysis, systematic review and RCT. It is also essential to widely implement continuing education and training for evaluation methodology of medicine safety, efficacy and economy. On the other hand, we should set up high quality evidence database and establish specialized evidence evaluation agencies [57]. These databases should be open to medical institutions, decision-making departments, and even the public for timely access to scientific evidence of medicines and other health technologies [58]. On the basis of availability of high quality evidences, concerned agencies will be likely to standardize the decision-making procedures for the inclusion of medicines into the NRDL, and formulate evidence evaluation methods and technical guidelines [59]. All these will facilitate the decision-making supervision and promote scientific and evidence-based decision-making.

Although this research and the findings are China-based, the policy implications probably have wider applicability in the sense that many other countries are also confronted with similar dilemma of decision-making while including medicines into NRDL. Even more important, this study provides a new strategy of comprehensively integrating international and local available high-grade evidence to make a rapid evaluation of NRDL's evidence-based rationality. Apparently, this strategy is not unique to a particular country or only applicable to single kinds of medicine. It can also be widely applied by many other countries to examine the evidence for certain kinds of medicines, such as antihypertensive medicines, anti-diabetic medicines, anti-cancer medicines and so on.

As a preliminary application of a rapid evaluation strategy, this study still has some limitations, which should be noticed when subsequently quoting the results or applying the evaluation strategy. Firstly, this study mainly collected published meta-analysis, systematic review, and RCT to evaluate the effectiveness of the medicines without direct evidence support from international evidence-based drug lists. However, the publication bias is still hard to be completely ruled out. Secondly, by considering scientific evidence support and representativeness, three international evidence-based medicine lists were selected as reference criteria in this study. However, there is still doubt about whether there are other international evidence-based medicine lists more appropriately for the reference criteria. In view of this issue, the intensive comparison between many commonly used international evidence-based medicine lists will be further considered in future studies. And the research outcomes will further improve the rapid evaluation strategy in this study.

\section{Conclusions}

Nearly $45 \%$ of the antihypertensive medicines included in the NRDL of UEBMI lack sound evidence supports for its effectiveness. Some shortcomings in reporting and methodological quality of published meta-analysis, systematic review, and RCT had deteriorated their authenticity and 
reliability, thus weakening their evidence support for certain antihypertensive medicines. It highlights the importance of providing professional and comprehensive methodological trainings to researchers and reinforcing methodological quality assurance in writing peer-reviewed journal articles. To optimize the selection of medicines into NRDL, it is recommended to establish specialized evidence evaluation agencies and set up high quality evidence database to timely provide sound evidence, as well as standardize the decision-making procedures for inclusion of medicines.

\section{Additional files}

Additional file 1: Table S1. The completion of each items in AMSTAR Scale_- - For the included systematic reviews and meta-analyses. It provides information on whether certain included systematic reviews and metaanalyses had completed each of the 11 items in AMSTAR Scale. (DOCX $27 \mathrm{~kb}$ )

Additional file 2: Table S2. Jadad score for the included randomized controlled trials. It provides information on whether certain included RCT had completed each of the 3 items, namely "randomization", "double blinding" and "withdrawals and dropouts", in the Jadad scale. (DOCX $21 \mathrm{~kb}$ )

\section{Abbreviations}

ACEls: Angiotensin Converting Enzyme Inhibitors; JNC 8: the Eighth Report of the Joint National Committee on Prevention, Detection, Evaluation and Treatment of high blood pressure; NICE 2011: the Clinical Management of Primary Hypertension in Adults, 2011 version; NRDL: National Reimbursement Drug List; UEBMI: Urban Employee Basic Medical Insurance; WHO EML 2017: World Health Organization Essential Medicine List of 2017 version

\section{Acknowledgements}

We gratefully acknowledge the funding support of China Medical Board (CMB 11-067), Fujian Education \& Scientific Research Project for Young Scholars (JA14140S) and the National Natural Science Foundation of China (71704026). And many thanks to Liangmei Lan for her great assistance on data extraction.

\section{Funding}

This study gained the funding support of China Medical Board (CMB 11-067), Fujian Education \& Scientific Research Project for Young Scholars (JA14140S) and the National Natural Science Foundation of China (71704026). And the funders had no involvement in study design, data collection, statistical analysis and manuscript writing.

\section{Availability of data and materials}

The datasets generated during and/or analyzed during the current study are available from the corresponding author on reasonable request.

\section{Authors' contributions}

YC and WL developed the research question and methodology. WL and XG developed the strategy of data collection and conduct the literature review. WL conducted the statistical analysis and developed the draft of manuscript. YC, MS and LS provided some guidance on data analysis and interpretation, and they also reviewed and revised the draft of manuscript. All five authors approved the final version of the manuscript.

\section{Ethics approval and consent to participate}

The research does not report on or involve the use of any animal or human data or tissue. Therefore, the ethics approval and consent to participate are not applicable.

\section{Consent for publication}

Not applicable.

\section{Competing interests}

The authors declare that they have no competing interests.

\section{Publisher's Note}

Springer Nature remains neutral with regard to jurisdictional claims in published maps and institutional affiliations.

\section{Author details}

${ }^{1}$ Department of Health Management, School of Public Health, Fujian Medical University, Fuzhou, Fujian, China. ${ }^{2}$ School of Public Health and Tropical Medicine, Tulane University, New Orleans, LA, USA. ${ }^{3}$ Department of Public Health Sciences, College of Health and Human Services, University of North Carolina at Charlotte, Charlotte, NC, USA. ${ }^{4}$ Department of Discipline Inspection, Children's Hospital, Nanjing Medical University, Nanjing, Jiangsu, China. ${ }^{5}$ Key Lab of Health Technology Assessment (Ministry of Health), Collaborative Innovation Center of Social Risks Governance in Health, School of Public Health, Fudan University, 446 Zhaojiabang Road Building 2 Room 1001, Xuhui District, Shanghai 200032, China.

Received: 24 October 2018 Accepted: 29 January 2019

Published online: 11 February 2019

\section{References}

1. Neumann PJ. Evidence-based and value-based formulary guidelines. Health Aff. 2004;23:124-34.

2. Yang $Y$, He P, Zou Y, Wang H, Wu Z. GRADE method and the application of it in the selection of essential drugs by WHO. China Pharm. 2011;22: 2977-80.

3. World Health Organization. The selection and use of essential medicines: report of the WHO expert committee. Geneva: World Health Organization; 2015.

4. Wang L, Yuan Q, Cheng L, Li Y. Reflection on the selection and evaluation of essential drug list. China Pharm. 2010;21:1453-6.

5. Fan L, Ma A. Discussion on the related questions about basic drug list selection. Health Economics Res. 2011:4:3-6.

6. Tian X, Song Y, Zhang X. National Essential Medicine List and policy practice: a case study of China's health care reform. BMC Health Serv Res. 2012;12:401

7. Guo Y, Gan L, Xing H, Yuan HM. Analysis on the successful experience of selecting essential drugs in the other countries. Chinese Pharm Aff. 2013;27:353-5.

8. Huang Y, Guo Y. Comparisons of the management models of domestic and foreign medical insurance covered medicine catalogs. Chinese Med Insur. 2013;12:68-70.

9. World Health Organization. WHO model list of essential medicines ( $20^{\text {th }}$ list). 2017. http://www.who.int/medicines/publications/essentialmedicines/en/. Assessed 22 May, 2017.

10. OgbaJoseph U, IheanyiDamian E. The eighth joint national committee on the prevention, detection, evaluation, and treatment of high blood pressure (joint national committee-8) report: Matters arising. Nigerian J Cardiol. 2017; 14:15. https://doi.org/10.4103/0189-7969.201909.

11. National Institute for Health and Clinical Excellence. Hypertension: Clinical Management of Primary Hypertension in Adults. NICE Clinical Guideline 127. London: National Clinical Guideline Centre; 2011.

12. Moher D, Liberati A, Tetzlaff J, Altman DG. The PRISMA Group. Preferred Reporting Items for Systematic Reviews and Meta-Analyses: The PRISMA Statement. PLoS Med. 2009;6(6):e1000097.

13. Shea BJ, Grimshaw JM, Wells GA, et al. Development of AMSTAR: a measurement tool to assess the methodological quality of systematic reviews. BMC Med Res Methodol. 2007;7:10.

14. Jadad AR, Moore RA, Carroll D, et al. Assessing the quality of reports of randomized clinical trials: is blinding necessary? Control Clin Trials. 1996;17:1-12.

15. Zeng X, Liu J, Chen J, et al. Efficacy and safety of Fosinopril for mild to moderate essential hypertension: a systematic review. China Pharm. 2014;25: $3372-6$.

16. Gasowski J, Wilkins A, Drzewoski J, et al. Short-term antihypertensive efficacy of perindopril according to clinical profile of 3,188 patients: a meta-analysis. Cardiol J. 2010;17(3):259-66. 
17. Chen S, Wang JJ, Wang QQ, et al. The effect of carvedilol and propranolol on portal hypertension in patients with cirrhosis: a meta-analysis. Patient Prefer Adherence. 2015;9:961-70.

18. Ke Z, Yao R, Chen Z, Fang C. Meta-analysis of the therapeutic efficacy and safety of doxazosin for mild to moderate essential hypertension. J Kunming Med Univ. 2015;36:28-32.

19. Zhou X, Ma W. Efficacy of Urapidil in treatment for heart failure with hypertension: a Meta-analysis. Chinese J Cardiovasc Res. 2016;14:735-8.

20. Li S, Qin L, Xu G. Effect of domestic Cilnidipine in the treatment of essential hypertension: a Meta analysis. Chinese Gen Pract. 2012;15:125-7.

21. Dong W, Huang T, Zhou Y, Dong Y. Effects of sodium nitroprusside in the treatment of hypertensive emergency: a systematic review. China Pharm. 2012;23:3778-82.

22. Hua Q. Meta-analysis of Lacidipine and amlodipine for essential hypertension. Front Med China. 2014;6:111-8.

23. Jiang C, Qiu J, Xu Y, et al. Meta analysis on Nicardipine in the treatment of hypertensive emergency. Chinese J Drug Appl Monit. 2013;10:245-50.

24. Ran Y, Wei L, Li D, Kuang F, Zhao C, Qian Y. The efficacy and tolerability of Lercanidipine and amlodipine for mild to moderate hypertension: Meta-analysis. Chongqing Med. 2015:44:4980-4.

25. Du B, Qin L, Cui W. Meta analysis on Arotinolol in treatment of essential hypertension. J Jilin Univ. 2009;35:949-54.

26. Zhao S, Yang W, Chen B. Meta-analysis of the efficacy and safety of benazepril vs. captopril in the treatment of primary hypertension. China Pharm. 2015:26:2524-6.

27. Zhang Y, Shou S, Liu Y, Wang L. The efficacy of Enopril folic acid in treatment of H type hypertension. Shandong Med. 2015:55:54-6.

28. Wu H, Xu G, Qin L. Meta-analysis on effectiveness and safety of irbesarten/ hydrochlorothiazide combination therapy in treatment of essential hypertension. J Jilin Univ (Medicine Edition). 2011;37:517-22.

29. Jin J, Yue Y, Jin Z, Wu F. Efficacy and safety of valsartan hydrochlorothiazide for primary hypertension: a Meta-analysis. Herald Med. 2013;32:394-7.

30. Zhao Z, Chen Y. Efficacy and safety of amlodipine vs. Levamlodipine for mild to moderate hypertension: a systematic review. Eval Anal Drug-Use Hosp China. 2015;15:318-21.

31. Chen Q, Ding D, Qin J. Efficacy of nimodipine combined with edaravone in treatment of hypertensive intracerebral hemorrhage: a meta-analysis. J Int Neurol Neurosurg. 2014;41:332-7.

32. Wu Y, He L, Song Y, Hu Y. Compound anti-hypertensive tablets (no.0) for primary hypertension: a systematic review. China J Dis Control Prev. 2009;13:245-50.

33. Magee LA, Namouz-Haddad S, Cao V, Koren G, Von DP. Labetalol for hypertension in pregnancy. Expert Opin Drug Saf. 2015;14(3):453-61.

34. Hu L, Liu S. Efficacy and safety of compound reserpine tablets for essential hypertension: systematic review. China Pharm. 2012;23:3219-23.

35. Garnockjones KP. Esmolol: a review of its use in the short-term treatment of tachyarrhythmias and the short-term control of tachycardia and hypertension. Drugs. 2012;72(1):109-32.

36. Du $X$, Yin $X$, Zhang $X$. Comprehensive evaluation on amlodipine and Nitrendipine therapy for hypertension. Chin Hosp Pharm J. 2014;34:59-62.

37. Zhang T, Wang X, Fan Q, Chen H, Hu Y. Efficacy and safety of amlodipine and Felodipine in the treatment of Chinese hypertension. PJCCPVD. 2013;21:37-40.

38. Kandler MR, Mah GT, Tejani AM, Stabler SN, Salzwedel DM. Hydralazine for essential hypertension. Cochrane Database Syst Rev. 2010;65(8):CD004934.

39. Shamon SD, Perez MI. Blood pressure lowering efficacy of reserpine for primary hypertension. Cochrane Database Syst Rev. 2009;202(4):CD007655.

40. Cao J, Lei J, Gong T, Li L, Chen G, Chen W. Antihypertensive efficacy of amlodipine benapril in the treatment of mild to moderate hypertension: a randomized controlled trial. Hebei Med J. 2012;34(7):1013-4.

41. Lu N, Lu K, Zhuang W, Xiang Q, Zhong Y, Ling J. The effectiveness and safety in treating unstable angina by metoprolol: a randomized controlled trial. Med Ind Inf. 2006:3(21):5-7.

42. Zuo W, Wang C, Xue X. Porspective study on the effects of drug intervention is preventing the first bleeding of esophageal varices: randomize controlled trial with propranolol, ISMN and spironolactone in combination. Chinese Hepatol. 2007:12(5):342-5.

43. Jiang $X$, Wang J, Chen S, Zhu C. A randomized controlled clinical trial in the prevention of esophageal variceal bleeding. Chin J Gastroenterol. 2001;6(2):90-3.

44. Huang $\mathrm{G}$, Jia J, Zhen Y, et al. Multicentric prospective randomized controlled study of efficacy of mannitol, furosemide and albumin in reducing intracranial pressure in patients with severe brain injury. Chin J Trauma. 2008;24(8):680-3.
45. Zheng W, Cao Y. Clinical randomized controlled trial on torasemide injection in treating patients with congestive heart failure and edema. Chin J Clin Pharmacol Ther. 2008;13(6):699-703.

46. Lu Q, Zhao M, Rong Y, Liu X. Random case-control study of the effectiveness of Naftopidil and terazosin in treatment of hypertension. Chin J Pharmacoepidemiol. 2000;9(1):1-3.

47. Liao Y. Randomized controlled trial on the efficacy of Olmesartan Medoxomil in remodeling left ventricular in treatment of morning surge of hypertension. Chin J Clin Ration Drug Use. 2014;7(4B):48-9.

48. Tao B, Guo J, Chen S, et al. Comparison of two combination regimens in the treatment of mild to moderate essential hypertension. Chinese J New Drugs. 2006;15:550-3.

49. Rokoss MJ, Teo KK. Ramipril in the treatment of vascular diseases. Expert Opin Pharmacother. 2005;6(11):1911-9.

50. Jiang $X$, Zhang $Y$, Wang $R$, et al. Comparison of imidapril and benazepril on central and peripheral blood pressures through pulse wave analysis. Chin J New Drugs Clin Rem. 2005:24:884-6.

51. Collaborative Group of Diltiazem. A multicenter, randomized clinical trial of intravenous diltiazem in treatment of unstable angina. Chin J Cardiol. 2005:33:238-42

52. Wang $X$, Ke $Y$, Wu W, Zhou $X$, Jiang S. Efficacy of combination of enalapril/ hydrochlorothiazide in Chinese patients with primary hypertension. Chin J New Drugs Clin Rem. 2006;25:759-62.

53. Schiffrin EL. Remodeling of resistance arteries in human hypertension: effects of Cilazapril, an angiotensin-l-converting enzyme inhibitor. Cardiology. 2008:86(S1):16-22

54. Li Y, Yu J, Du L, et al. Exploration and practice of methods and processes of evidence-based rapid review on peer review of WHO EML application. Chin J Evid Based Med. 2015;15:1447-53.

55. Chen Y, Banta D, Tang Z. Health technology assessment development in China. Int J Technol Assess Health Care. 2009;25(Suppl 1):S202-9.

56. Liu W, Shi L, Pong RW, et al. Differences in evaluating health technology assessment knowledge translation by researchers and policy-makers in China. Int J Technol Assess Health Care. 2014;30:612-20.

57. Cui M, Peng Y, Zhao J, Fang T. Comparative study of the health insurance directory entrance procedure of new drug domestic and overseas and the suggestion on the relevant policy in China. J Pharm Pract. 2011;29:226-8.

58. Laupacis A. Economic evaluations in the Canadian common drug review. Pharmacoeconomics. 2006:24:1157.

59. Daniels N, Teagarden JR, Sabin JE. An ethical template for pharmacy benefits. Health Aff. 2003:22:125.

Ready to submit your research? Choose BMC and benefit from

- fast, convenient online submission

- thorough peer review by experienced researchers in your field

- rapid publication on acceptance

- support for research data, including large and complex data types

- gold Open Access which fosters wider collaboration and increased citations

- maximum visibility for your research: over $100 \mathrm{M}$ website views per year

At $\mathrm{BMC}$, research is always in progress.

Learn more biomedcentral.com/submission 